Corrigendum

\title{
Corrigendum to "Niemann-Pick Disease Type C Presenting as a Developmental Coordination Disorder with Bullying by Peers in a School-Age Child"
}

\author{
Ryo Suzuki, ${ }^{1}$ Atsushi Tanaka, ${ }^{1}$ Toshiharu Matsui, ${ }^{1}$ Tetsuki Gunji, ${ }^{1}$ Jun Tohyama, ${ }^{2}$ \\ Aya Narita, ${ }^{3}$ Eiji Nanba, ${ }^{4}$ and Kousaku Ohno ${ }^{5}$ \\ ${ }^{1}$ Department of Pediatrics, Nagaoka Chuo General Hospital, 2041 Kawasaki-cho, Nagaoka, Niigata 940-8653, Japan \\ ${ }^{2}$ Department of Child Neurology, Nishi-Niigata Chuo National Hospital, 1-14-1 Masago, Nishi-ku, Niigata, Niigata 950-2085, Japan \\ ${ }^{3}$ Division of Child Neurology, Institute of Neurological Sciences, Faculty of Medicine, Tottori University, 36-1 Nishimachi, \\ Yonago, Tottori 683-8504, Japan \\ ${ }^{4}$ Division of Functional Genomics, Research Center for Bioscience and Technology, Tottori University, 86 Nishimachi, Yonago, \\ Tottori 683-8503, Japan \\ ${ }^{5}$ Sanin Rosai Hospital, 1-8-1 Kaike Shinden, Yonago, Tottori 683-8605, Japan \\ Correspondence should be addressed to Ryo Suzuki; rsuzuki-tym@umin.ac.jp
}

Received 17 July 2017; Accepted 27 July 2017; Published 20 September 2017

Copyright (C) 2017 Ryo Suzuki et al. This is an open access article distributed under the Creative Commons Attribution License, which permits unrestricted use, distribution, and reproduction in any medium, provided the original work is properly cited.

In the article titled "Niemann-Pick Disease Type C Presenting as a Developmental Coordination Disorder with Bullying by Peers in a School-Age Child" [1], the name of the Sixth author was given incorrectly as Aya Nairita. The author's name should have been written as Aya Narita. The revised authors' list is shown above.

\section{References}

[1] R. Suzuki, A. Tanaka, T. Matsui et al., "Niemann-pick disease type $\mathrm{C}$ presenting as a developmental coordination disorder with bullying by peers in a school-age child," Case Reports in Pediatrics, vol. 2015, Article ID 807591, 4 pages, 2015. 


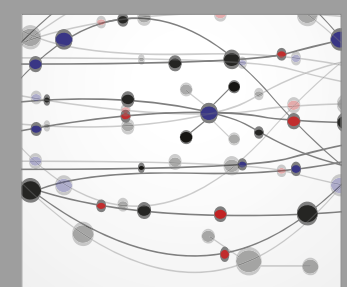

The Scientific World Journal
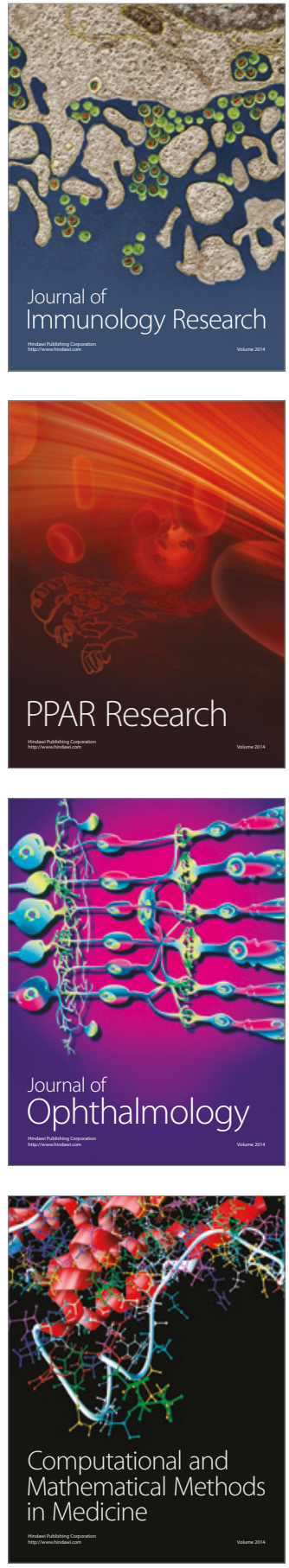

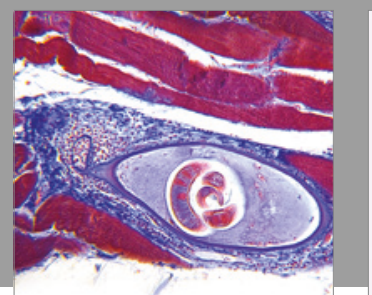

Gastroenterology Research and Practice
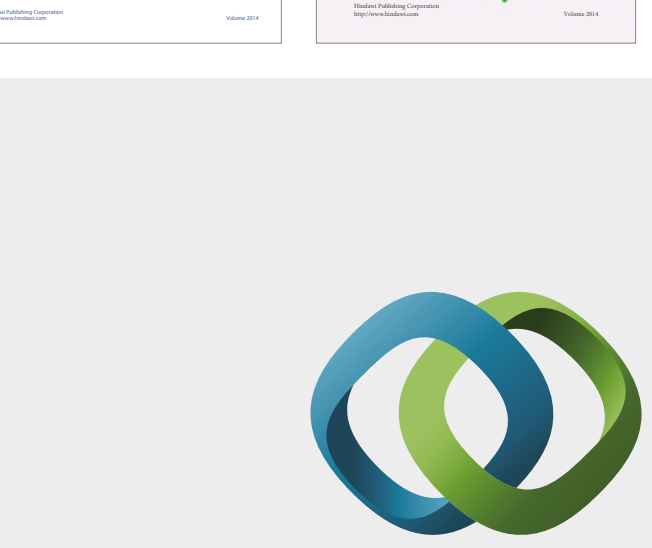

\section{Hindawi}

Submit your manuscripts at

https://www.hindawi.com
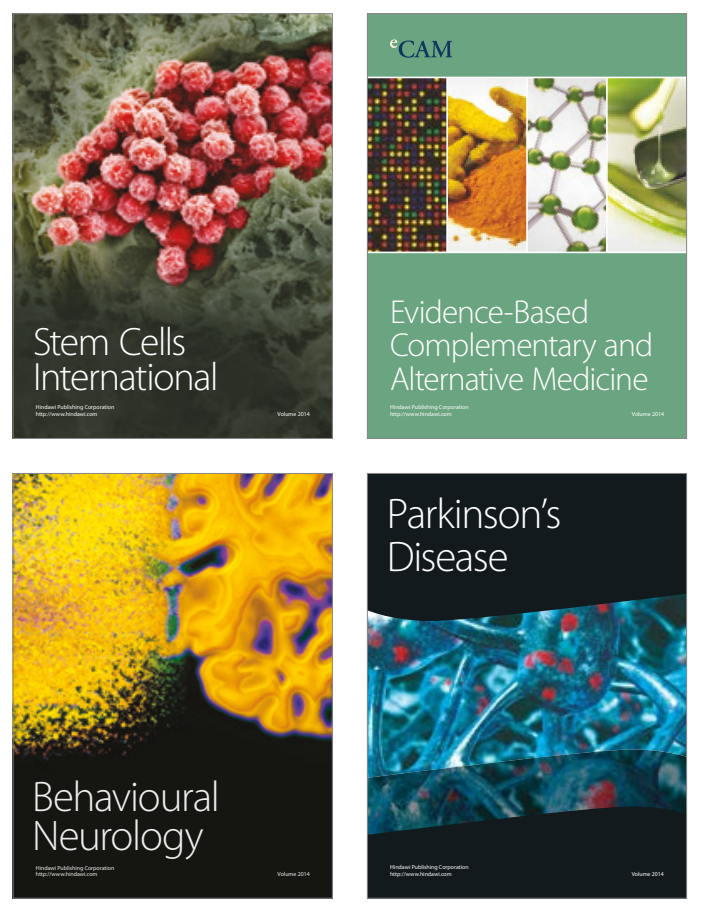
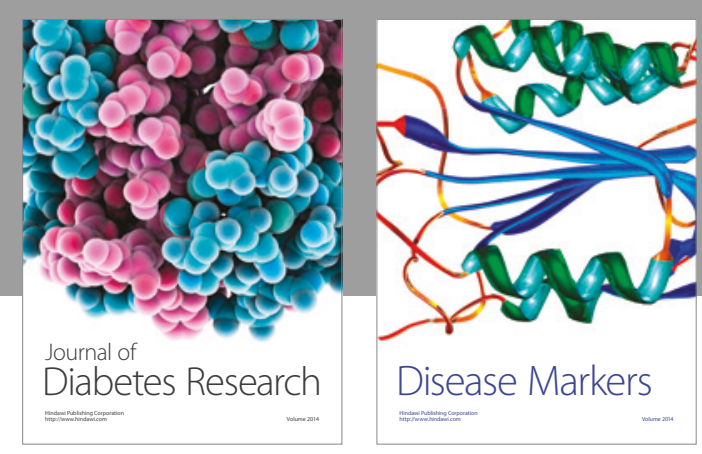

Disease Markers
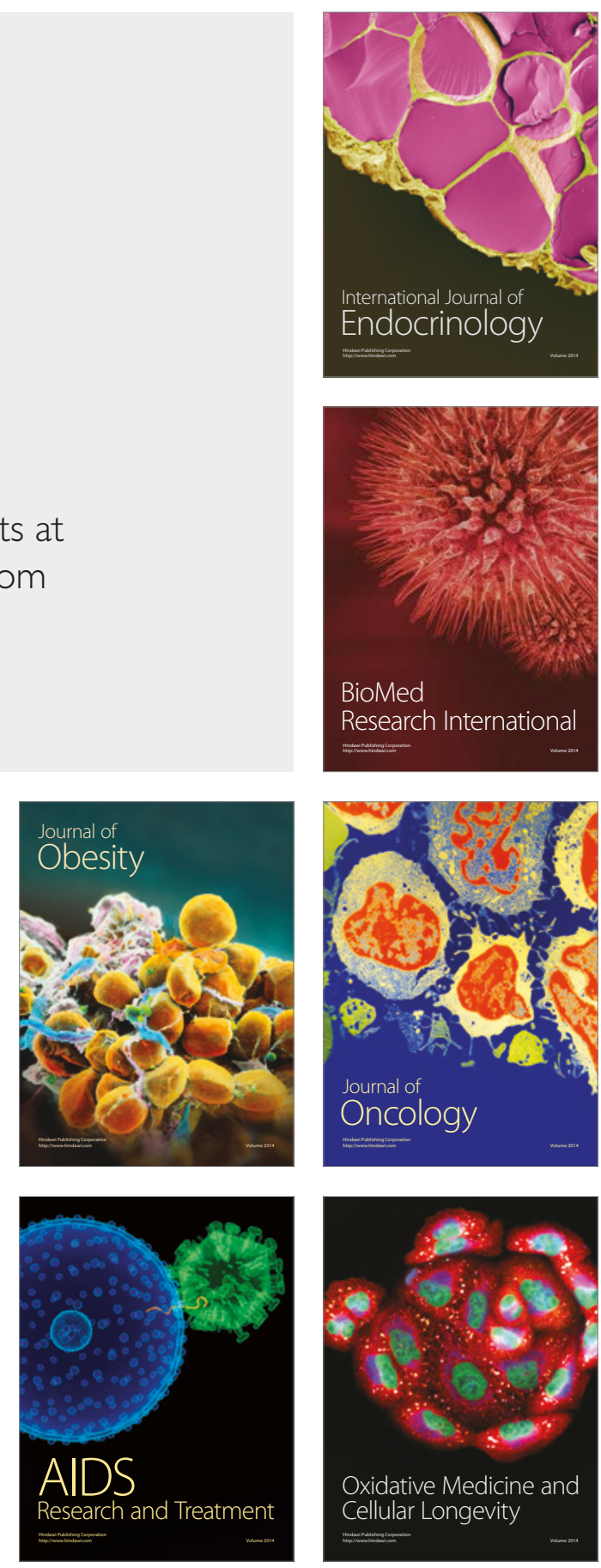